\title{
The Research of Contrast Enhancement Algorithm in Laser Projection Display System
}

\author{
Bailin Na, Yingying Wu \\ School of Information Science and Technology, East China Normal University, Shanghai, China \\ Email: smartfour@126.com
}

Received September 30, 2012; revised October 29, 2012; accepted November 10, 2012

\begin{abstract}
High-contrast is one of the main advantages in laser projection display, and the method of DCC (Dynamic Contrast Control) is the main way to increase the contrast. Generally, image pre-processing is necessary for eliminating noise and decreasing the over-highlight. In this paper, we proposed and actualized a method by following 3 steps: Firstly, the original image was analyzed statistically to get the scope of gray-scale distribution and average gray-scale; and then the image was divided into a number of sub-images. The sub-images whose pixels are higher than a certain threshold in both number and range, are applied image segmentation by certain growth rules. The sub-images satisfied with the growth rules are marked 1 , and the rests are marked 0 . Secondly, the sub-images are uniting. A sub-image has 3 relations between 8 sub-images around it: 1 and 1,1 and 0,0 and 0 . The sub-images marked 1 are uniting together, and the sub-images marked 0 are uniting together. Without affecting the visual vision, all over-highlight pixels were reduced in a certain proportion. Lastly, based on the application of DCC, the whole image signals were enlarged and the brightness of light sources were reduced, so as to achieve the desired effect in contrast enhancement.
\end{abstract}

Keywords: Contrast; Region-Grow Segmentation; Edge-Tracking

\section{Introduction}

Liquid crystal on silicon (LCOS) is a "micro-projection" or "micro-display" technology typically applied in projection televisions. It is a reflective technology similar to DLP projectors; however, it uses liquid crystals instead of individual mirrors. By way of comparison, LCD projectors use transmissive LCD chips, allowing light to pass through the liquid crystal. In LCOS, liquid crystals are applied directly to the surface of a silicon chip coated with an aluminized layer, with some type of passivation layer, which is highly reflective.

A key metric of image quality for a projected or directly-viewed image is the contrast ratio. Contrast ratio itself consists of two measurements, "on/off contrast", or full-screen contrast, and ANSI contrast which uses a field of 16 black and white rectangles. ANSI contrast can be used to describe the influence of light scattering on the display, and ANSI contrast cannot exceed the value of full-screen contrast. For computer graphics displays, where images have large areas of white or other bright colors displayed, the ANSI contrast value is a very useful metric. An ANSI contrast value of 300:1 is usually considered sufficient due to the dynamic range limitations of the human eye.

\section{Projection Display Principle}

In the projection display system, the mechanism of projection display can be equivalent to the formula:

$$
M=S * I
$$

where $M$ stands for the final image projected on the screen, $S$ stands for the signal intensity of image, and $I$ stands for the luminance of light source. In conventional systems, the DCC is used to enhance the contrast to improve the image visual effects. The DCC increase the signal intensity, and reduce the luminance of light source simultaneously. So the interferences of parasitic light and diffractive light are also be reduced too. Just as the formula:

$$
M=(\partial S) *\left(\frac{1}{\partial} I\right)(\partial>1) .
$$

Histogram modification based algorithm is the most popular approaches to achieve widely dynamic range. Histogram Equalization (HE) is one of the most commonly used algorithms to perform contrast enhancement due to its simplicity and effectiveness. In general, the HE distributes pixel values uniformly and results in enhanced images with linear cumulative histogram. But there are many disadvantages that $\mathrm{HE}$ enhances the entire image pixels and the visual results are hard to control, and most 
of all is that the noises are enhanced too.

So in this paper, we proposed and actualized a new system based on the principle of image segmentation.

\section{Image Segmentation}

Image segmentation is one of the most important tasks in image processing, and has a wide range of applications in computer vision, such as pattern recognition, image compression and so on. The image segmentation approaches can be divided into four categories: thresholding, clustering, edge detection and region extraction. In this paper, a region extraction based method for image segmentation will be considered, the special regions which has a strongly contrast to its surroundings will be gotten by using image segmentation [1].

We detect the edges firstly by using the templates which have $(2 \mathrm{P}+1) *(2 \mathrm{P}+1)$ pixels in size. If the center pixel of the template is located in non-edge regions, then the whole gray-scale changes within the template should be gentler. In other words, the gray value of center pixel should be close to the pixels which are located around it. If the center pixel in the template is located in edge region, then the gray-scale changes in the template region should be relatively intense.

To achieve the above purposes, we definite a variable $\gamma$ to reflect the standard deviation of the changes in local regions of gray-scale image, for a given image $g$ $(i, j)$. With the local gray-scale change from moderate to severe, the $\gamma$ would increase in correspond [2]. We use the template $P$ to mean-filter the whole image, to calcu- late the mean of local pixels $\left(m_{g}\right)$, and the standard deviation of local pixels $\left(\gamma_{\partial}\right)$ by the formulas:

$$
\begin{aligned}
& m_{g}(i, j)=\frac{1}{(2 p+1)^{2}} \sum_{k=i-p}^{i+p} \sum_{l=j-p}^{j+p} g(k, l) \\
& \gamma_{\partial}(i, j)=\sqrt{\frac{1}{(2 p+1)^{2}} \sum_{k=i-p}^{i+p} \sum_{l=j-p}^{j+p}\left[g(k, l)-m_{g}(k, l)\right]^{2}}
\end{aligned}
$$

Figure 1(a) is the original image, and the Figure 1(b) is processed image. In Figure 1(b), we can see the obvious edge of local region and noise. So the next step, we use Gauss Filter and Median Filter to eliminate the disturbances of noises. After that we need to choose a threshold which is very important in the step to keep the high contrast edges and ignore the low contrast edges. In this paper, a large number of images are analyzed statistically to get the scope of the threshold in that the choice of the threshold will be good to eliminate interference. The image below has been processed with the threshold.

Figure 2(b) is the processed image. We can see that most of noises are eliminated and the high-contrast edges are strengthened. The next step we need to estimate whether that there are closed regions in the image or not. If there are closed regions, then we continue to work. If not, we do not process the image anymore. We estimate it in this way:

The method to estimate closed regions is edge-tracking. We use counterclockwise edge-tracking in accordance with the order of the arrows in Figure 3(a). At the same time; we mark the pixels every time to prevent in-

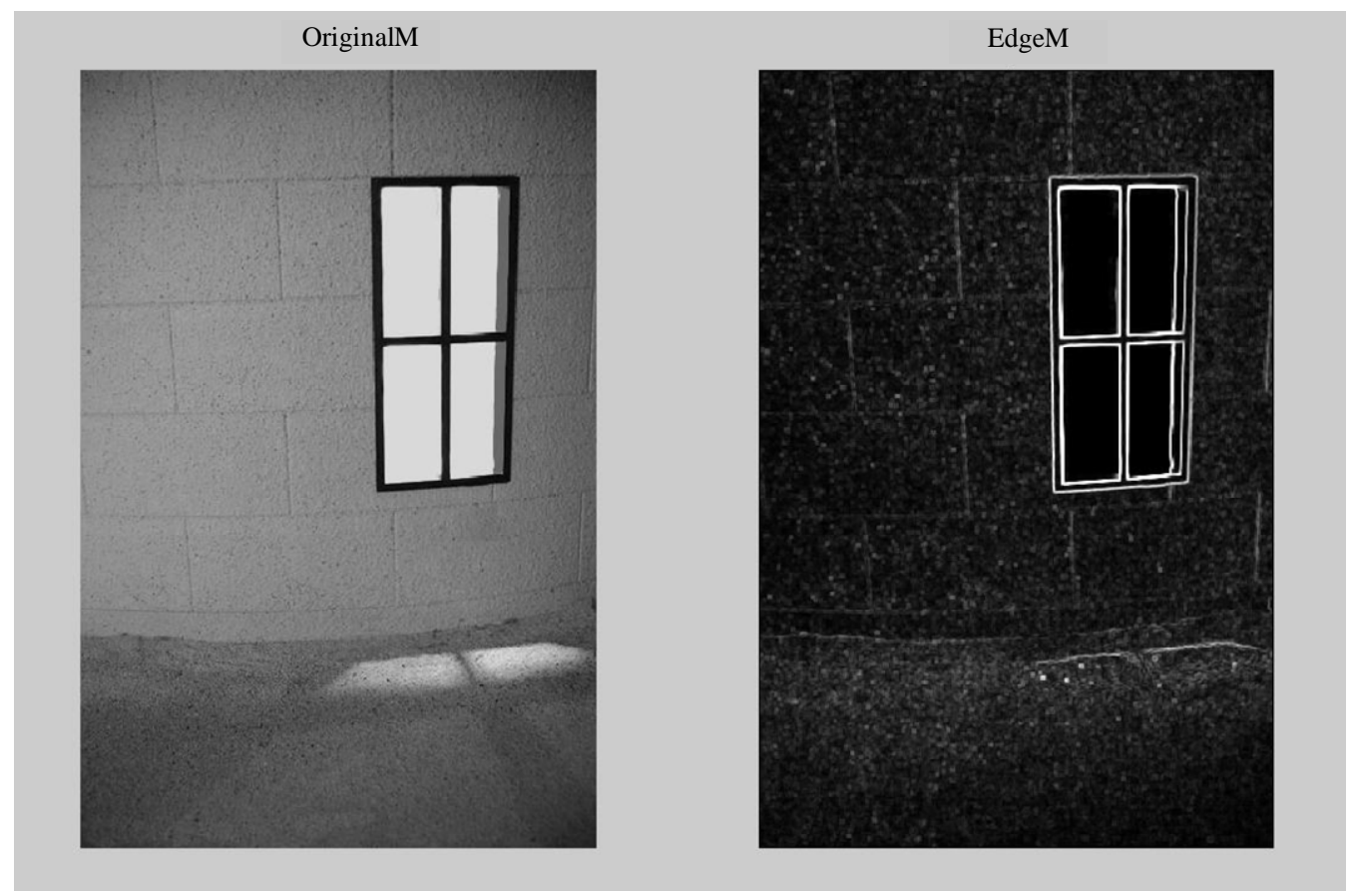

Figure 1. The original image and processed image. 


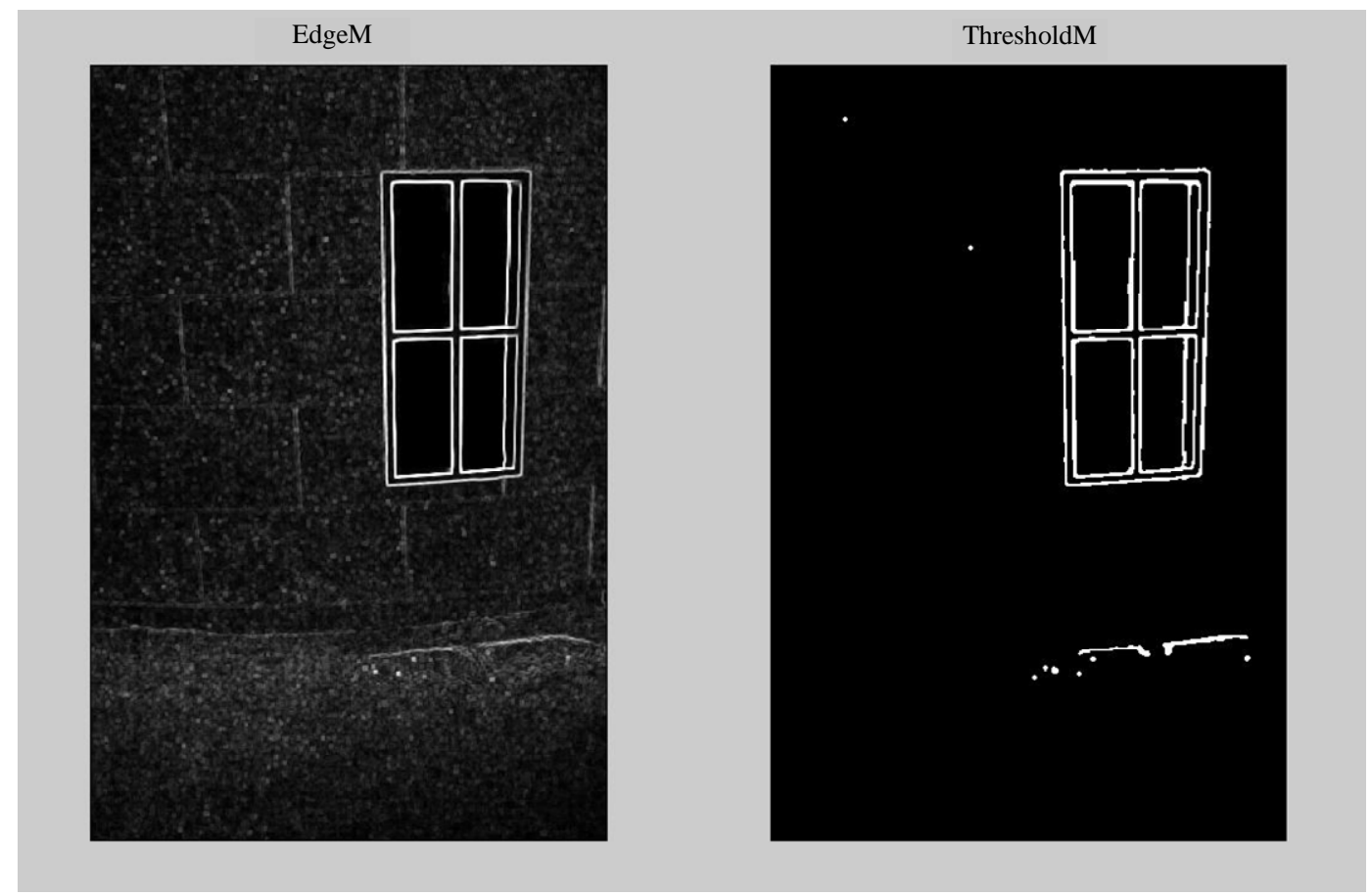

Figure 2. The edge image.

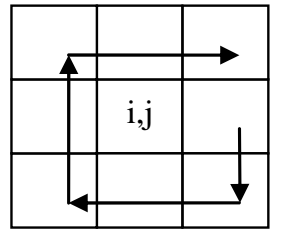

\begin{tabular}{|l|l|l|}
\hline 6 & 7 & 8 \\
\hline 5 & $\mathrm{i}, \mathrm{j}$ & 1 \\
\hline 4 & 3 & 2 \\
\hline
\end{tabular}

Figure 3. Growth rule.

finite loops. We track the edge according to the direction of the arrow. Part of the code is as follows:

$$
\begin{aligned}
& \text { if }(M(i, j+1)=\text { threshold }) \&(\text { State } !=5) \\
& \text { mask }(i, j+1)=1 ; \\
& i=i+1 \\
& j=j+1
\end{aligned}
$$$$
\text { State }=1 \text {. }
$$

\section{Region-Growing}

Region-growing approaches exploit the important fact that the pixels which are close together have similar gray values. In region-growing process, there are two factors must to be considered, the first one is how to choose the seed(s) in practice, and the second one is how to choose the similarity criteria. The method of selecting the seeds is a key step to segmentation, because the segment result is sensitive to the selection of the initial growing points. For example, the result of region growing will go awry if the initial seed falls on a noise point. For a good segmentation, it is required that the regions have relative uniform gray value and the seed pixels have a gray value which is typical of the region. In this paper, we will make some improvement on the method of selecting seeds and region growing rules [3].

The method we proposed:

1) We analyze the entire image pixels statistically, and then calculate the average gray of the image. In this step, we need to get the threshold which can be calculated based on adaptive algorithm, and the threshold would be applied in the next steps.

2) We divide the original image into a number of sub-images which are in the same size. (The number of sub-images would affect the image segmentation). The gray-scale of the sub-images is analyzed statistically. The pixels which are greater than the threshold are called high-light pixels, and the number of high-light pixels of every sub-image is counted. If the high-light pixels number of a sub-image is larger than the stipulated limit, the sub-image should be marked 1 , and process the regiongrowing in the next step. If not, the sub-image should be marked 0, do nothing [4].

3) The sub-images marked 1 are processed with region-growing algorithm.

The seeds of region-growing: we analyze the sub-image statically to get the seeds.

The rules of region-growing: the seeds grow anticlockwise among the 8 pixels around it.

4) The last step was region uniting. A sub-image has 3 relations between 8 sub-images around it: 1 and 1, 1 and 0,0 and 0 . So the relationship between the sub-images could be concluded in three kinds. In the first two kinds of situation, the relationship between the pixels at the 
sub-image edge is used to determine whether to uniting or not. In this step, if the sub-images which are marked 0 have been uniting with others, they would be marked 1 after uniting. Finally, in the kind of 00 , if the pixels at the edge between two sub-images meet the growth rules, the two sub-images are uniting, and are marked 1 . The sub-images which are still marked 0 after segmentation, are considered as noise and would be eliminated

Figure 4(a) is the effect image after image segmenta- tion.

\section{Result and Conclusions}

To compare and evaluate the processing result, we do some experiments on general photo image.

Figure 5(a) is the original image. Figure 5(b) is processed image. Without affecting the visual vision, the regions of all over-highlight pixels were reduced in a certain proportion.

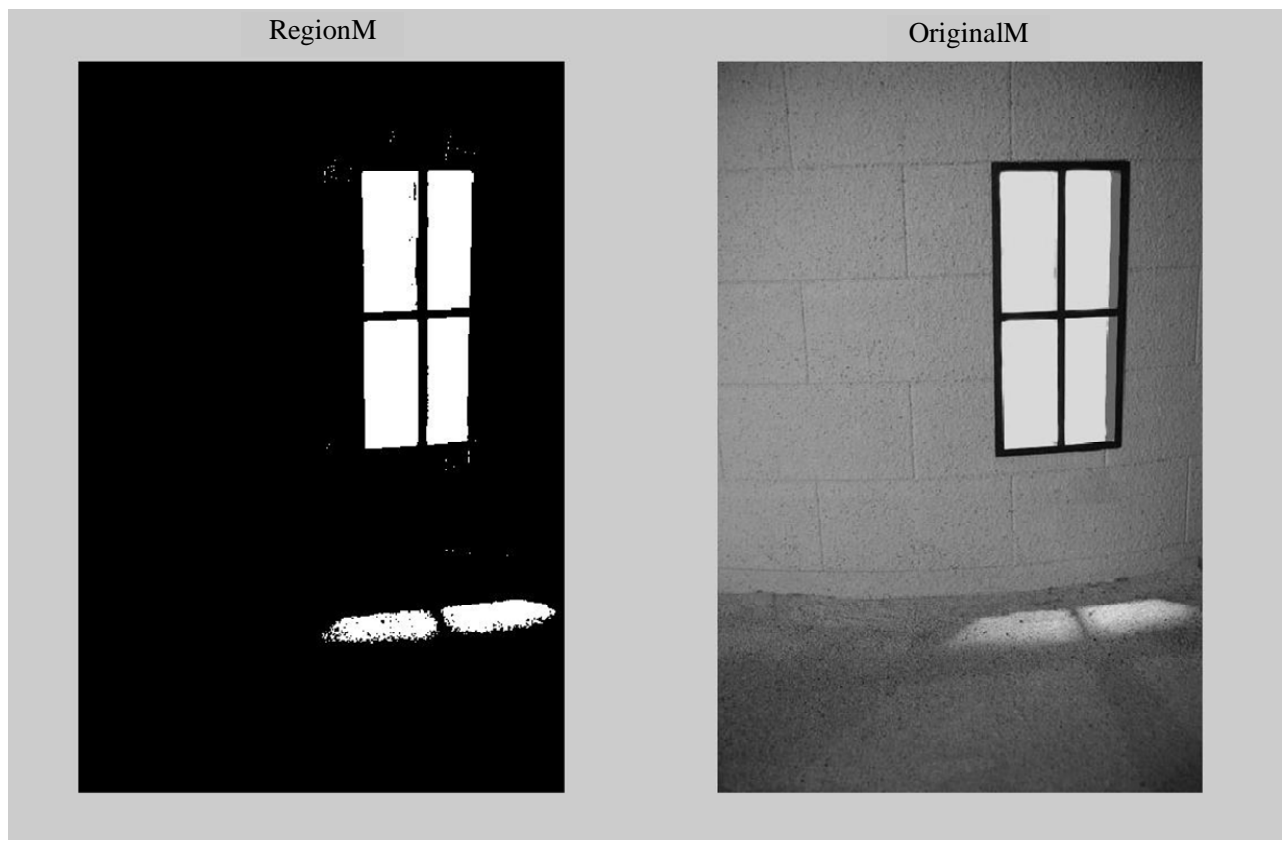

Figure 4. The highlight areas.

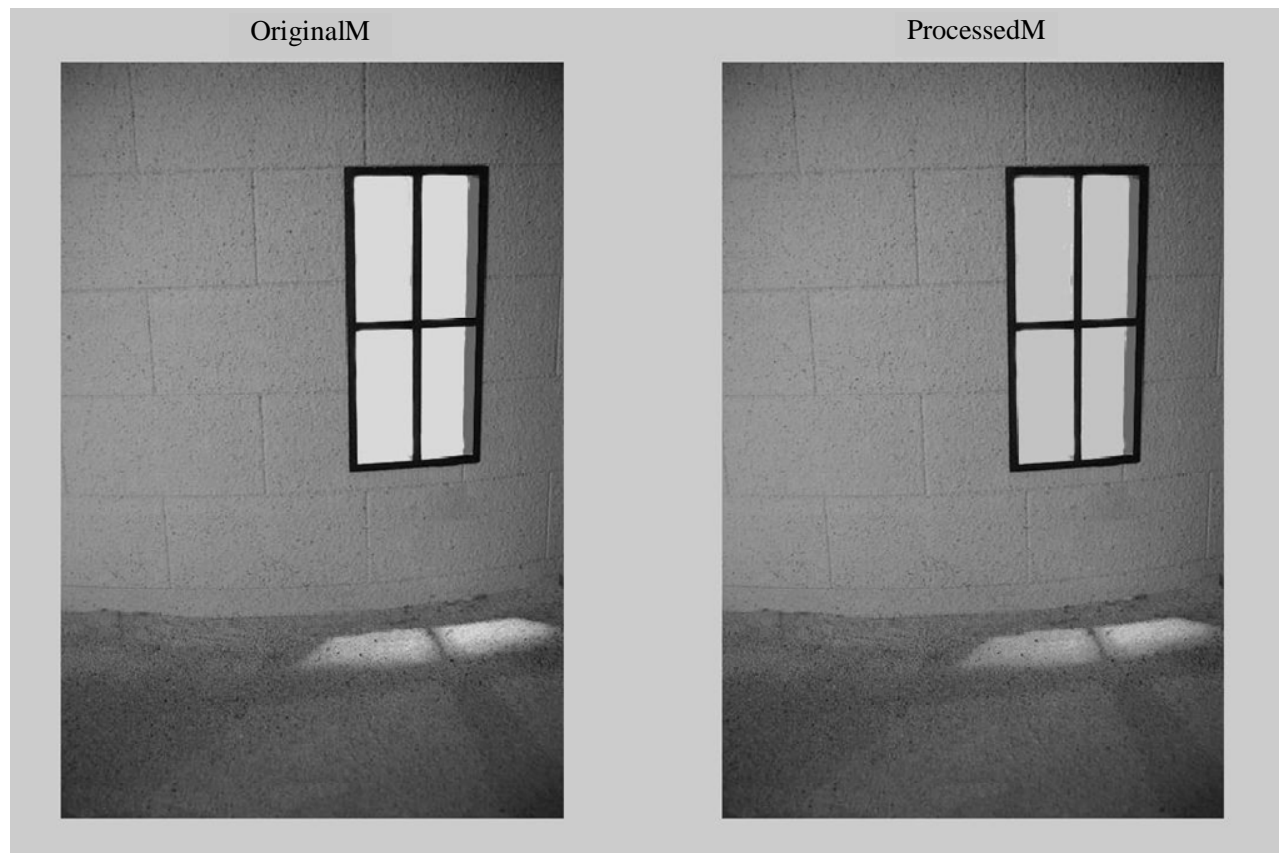

Figure 5. The original image and the final image. 


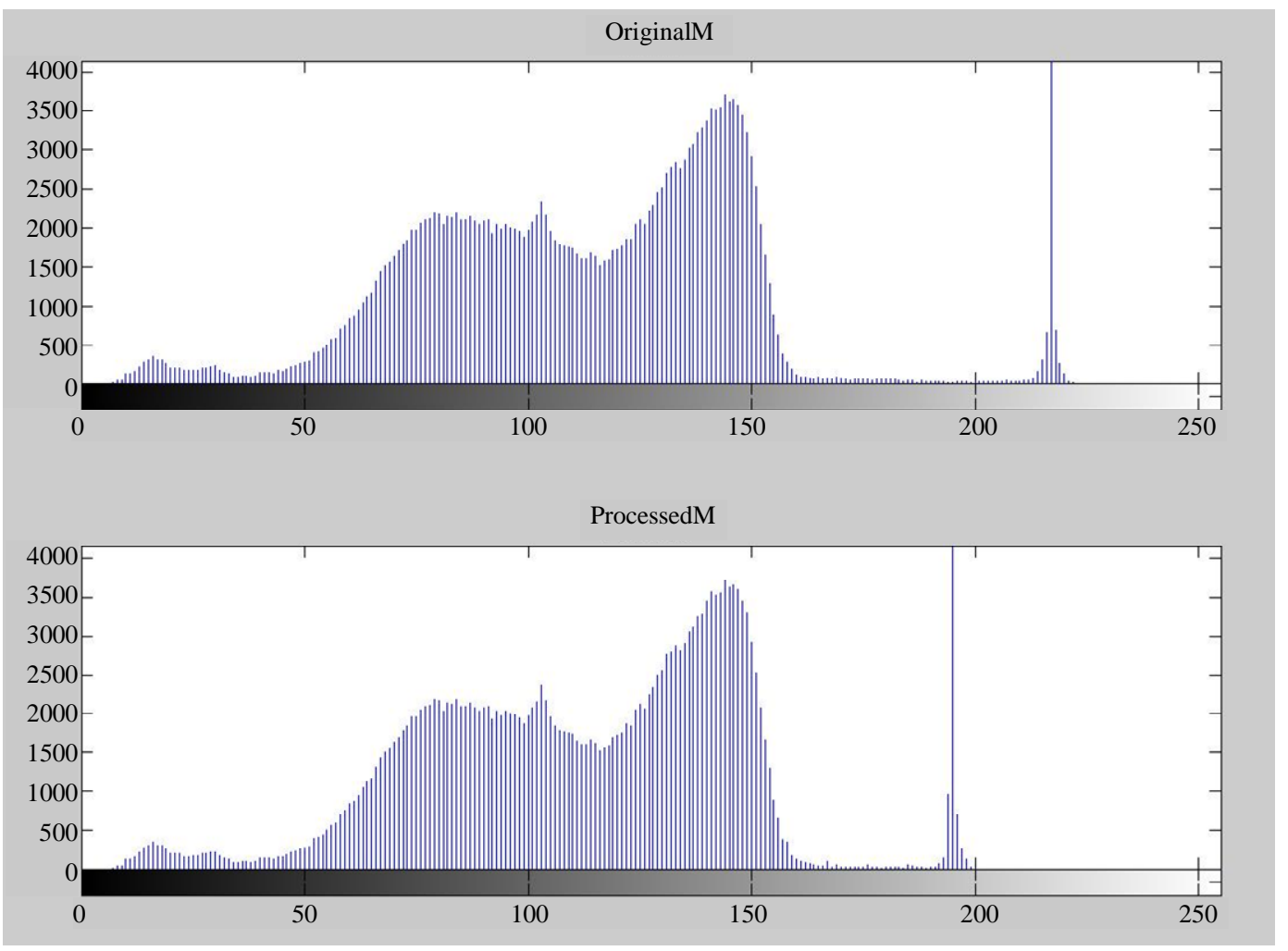

Figure 6. The HEs of the original image and the final image.

Figures 6(a) and (b) are the histograms of the original image and the processed image. We can see that the main parts of the two wave shapes have not changed, that means the backgrounds of the image stay the same. And the waveform of the highlight regions have not changed but moved, that means the highlight regions have been reduced. For example: If the original image could be enlarged 1.2 times, then the processed could be enlarged 1.3 times:

$$
M=(1.3 * S) *\left(\frac{1}{1.3} I\right)
$$

Lastly with the application of DCC, the whole image signal was enlarged and brightness of light source was reduced, so as to achieve the desired effect in contrast enhancement.

\section{Acknowledgements}

Our research is supported by the National High Tech Research and Development Program of China (2007AA-
030112 and 2009AA032708).

\section{REFERENCES}

[1] S.-Y. Wan and E. H. William, "Symmetric Region Growing," IEEE Transactions on Image Processing, Vol. 12, No. 9, 2003, pp. 1007-1015. doi:10.1109/TIP.2003.815258

[2] Y. S. Frank and S. X. Cheng, "Automatic Seeded Region Growing for Color Image Segmentation,” Image and Vision Computing, Vol. 23, No. 10, 2005, pp. 877-886. doi:10.1016/j.imavis.2005.05.015

[3] L. T. Law and Y. M. Cheung, "Color Image Segmentation Using Rival Penalized Controlled Competitive Learning," Proceedings of the International Joint Conference on Neural Networks, IJCNN'03, Portland, 20-24 July 2003, pp. 108-112. doi:10.1109/IJCNN.2003.1223306

[4] R. Pohle and K. D. Toennies, "Segmentation of Medical Images Using Adaptive Region Growing," Proceedings of SPIE-The International Society for Optical Engineering, Vol. 4322, No. 3, 2001, pp. 1337-1346. 